\title{
Entropy production and mesh refinement - application to wave breaking
}

\author{
L. Yushchenko a, F. Golay and M. Ersoy \\ Université de Toulon, IMATH, EA 2134, 83957 La Garde Cedex, France
}

Received 18 June 2013, Accepted 17 January 2015

\begin{abstract}
We propose an adaptive numerical scheme for hyperbolic conservation laws based on the numerical density of entropy production (the amount of violation of the theoretical entropy inequality). Thus it is used as an a posteriori error which provides information on the need to refine the mesh in the regions where discontinuities occur and to coarsen the mesh in the regions where the solutions remain smooth. Nevertheless, due to the CFL stability condition the time step is restricted and leads to time consuming simulations. Therefore, we propose a local time stepping algorithm. This approach is validated in the case of classical 1D numerical tests. Then, we present a 3D application. Indeed, according to an eulerian bi-fluid formulation at low Mach, an hyperbolic system of conservation laws allows an easily parallelization and mesh refinement by "blocks".
\end{abstract}

Key words: Hyperbolic systems / finite volume / local mesh refinement / numerical density of entropy production / local time stepping / wave breaking

\section{Introduction}

The present study deals with the numerical simulation of multi-fluid flows such as wave propagating, wave breaking and impacting on structure. Numerical simulations of these processes generally involve the complete resolution of the Navier-Stokes equations in both air and water phases. This approach is widely used for its physical relevancy, but its cost in terms of cpu-time remains a major drawback. Three-dimensional simulations still require a significant effort in software development and mesh refinement technique on powerful computers [1-6]. Simplified models based on potential theory or shallowwater equations [7-9] can be used to reduce the cpu-time. Unfortunately, they are not able to adequately represent the complex free surface dynamics occurring during wave breaking. An alternative intermediate formulation is used here, aiming to be physically more relevant than shallowwater models and less cpu-time consuming than the full Navier-Stokes simulations. The model is based on a fast 3D two phases flow solver: viscous effects are neglected and an artificial compressibility approach is used leading to an hyperbolic system of conservation laws. This formulation, combined with explicit time integration, allows an efficient parallel implementation. Our model has been successively validated on experimental and numerical test

${ }^{a}$ Corresponding author: yushchen@univ-tln.fr cases [10], improved by the selection of an isothermal model [11] and recently tested on breaking wave problem over a sloping bottom with and without roughness elements [12]. In order to keep accurate simulation in a reasonable cpu-time, it is relevant to use adaptive mesh refinement technique based on an efficient criterion. Recent developments proved that the numerical production of entropy is a very powerful error "like" indicator [13-16]. This approach leads to a super-convergent scheme [17]. Coupled to a local time stepping scheme, it allows to significantly reduce the computational time. The method is recalled in the first part of the paper. The second part concerns the numerical validation in the case of one dimensional test. Application on a wave breaking problem is shown in the third part while the last section is dedicated to conclusion and prospects.

\section{Entropy production}

We are interested in numerical integration of non linear hyperbolic systems of conservation laws of the form (express here in $1 \mathrm{~d}$ for the sake of simplicity).

$$
\left\{\begin{array}{l}
\frac{\partial \mathbf{w}}{\partial t}+\frac{\partial \mathbf{f}(\mathbf{w})}{\partial x}=0, \quad(t, x) \in \mathbb{R}^{+} \times \mathbb{R} \\
\mathbf{w}(0, x)=\mathbf{w}_{0}(x), \quad x \in \mathbb{R} .
\end{array}\right.
$$


L. Yushchenko et al.: Mechanics \& Industry 16, 301 (2015)

Table 1. Comparison of numerical schemes of order 1 and 2.

\begin{tabular}{cccccc}
\hline & $\mathcal{P}$ & $\left\|\rho-\rho_{\text {ref }}\right\|_{l_{x}^{1}}$ & cpu-time & $N_{L_{\max }}$ & $\begin{array}{c}\text { Maximum number } \\
\text { of cells }\end{array}$ \\
\hline AB1 & 0.288 & 0.0474 & 181 & 1574 & 2308 \\
AB1M & 0.288 & 0.0480 & 120 & 1572 & 2314 \\
AB2 & 0.287 & 0.0275 & 170 & 1391 & 2023 \\
AB2M & 0.286 & 0.0274 & 108 & 1357 & 1994 \\
RK2 & 0.285 & 0.0208 & 299 & 1375 & 2005 \\
\hline
\end{tabular}

where $\mathbf{w}: \mathbb{R}^{+} \times \mathbb{R} \rightarrow \mathbb{R}^{d}$ stands for the vector state and $\mathbf{f}: \mathbb{R}^{d} \rightarrow \mathbb{R}^{d}$ the flux function.

Solving Equation (1) with high accuracy is a challenging problem since it is well-known that solutions can and will breakdown at a finite time, even if the initial data are smooth, and develop complex structure (shock wave interactions). In such a situation, the uniqueness of the (weak) solution is lost and is recovered by completing the system (1) with an entropy inequality of the form:

$$
\frac{\partial s(\mathbf{w})}{\partial t}+\frac{\partial \psi(\mathbf{w})}{\partial x} \leqslant 0
$$

where $(s, \psi)$ stands for a convex entropy-entropy flux pair. This inequality allows to select the physical relevant solution. Moreover, the entropy satisfies a conservation equation only in regions where the solution is smooth and an inequality when the solution develops shocks. In simple cases, it can be proved that the term missing in (2) to make it an equality is a Dirac mass.

Numerical approximation of Equations (1) with (2) leads to the so-called numerical density of entropy production, which is a measure of the amount of violation of the entropy equation (as a measure of the local residual as in [18-20]). As a consequence, the numerical density of entropy production provides information on the need to locally refine the mesh (e.g. if the solution develops discontinuities) or to coarsen the mesh (e.g. if the solution is smooth and well-approximated) as already used by Puppo [14-16] and Golay [13]. Even if the shocks are well-captured on coarse grid using finite volume scheme, such indicator is able (as shown in Puppo [16]) not only to provide an efficient a posteriori error, but also to reproduce the qualitative structure of the solution and to pilot the adaptive scheme. Explicit adaptive schemes are well-known to be time consuming due to a CFL stability condition. The cpu-time increases rapidly as the mesh is refined. Nevertheless, the cpu-time can be significantly reduced using the local time stepping algorithm (see e.g. [17, 21-24]).

The numerical scheme is presented in details in [17], including the local time stepping scheme, the mesh refinement procedure by dyadic tree. The reader can found more details about the $3 \mathrm{~d}$ approach in $[11,12]$.

\section{One-dimensional test case}

We now present some results using the adaptive multi scale scheme. Numerical solutions are computed using the one-dimensional gas dynamics equations for ideal gas:

$$
\begin{aligned}
& \frac{\partial \rho}{\partial t}+\frac{\partial \rho u}{\partial x}=0, \quad \frac{\partial \rho u}{\partial t}+\frac{\partial\left(\rho u^{2}+p\right)}{\partial x}=0, \\
& \frac{\partial \rho E}{\partial t}+\frac{\partial(\rho E+p) u}{\partial x}=0, \quad p=(\gamma-1) \rho \varepsilon
\end{aligned}
$$

where $\rho, u, p, \gamma, E$ are respectively the density, the velocity, the pressure, the ratio of the specific heats (set to 1.4 ) and the total energy $E=\varepsilon+u^{2} / 2$ (where $\varepsilon$ is the internal specific energy). Using the conservative variables $\mathbf{w}=(\rho, \rho u, \rho E)^{T}$, we classically define the entropy by $s(\mathbf{w})=-\rho \ln \left(p / \rho^{\gamma}\right)$ and the entropy flux by $\psi(\mathbf{w})=u s(\mathbf{w})$.

In what follows, we perform several numerical tests. We will refer AB1 as the first order scheme, AB2 as the second order Adams-Basforth scheme, RK2 as the second order Runge-Kutta scheme. AB2 and RK2 use a MUSCL reconstruction. Moreover, all computations are made with a dynamic grid. We also use the cap "M" when the local time stepping algorithm is employed. $N_{L_{\max }}$ stands for the average number of cells used during a simulation of an adaptive mesh refinement scheme with a maximum level $L_{\max }$.

We consider the Shu and Osher's problem [25] with initial conditions

$(\rho, u, p)(0, x)= \begin{cases}(3.857143,2.629369,10.3333), & x \leq 0.1 \\ (1+0.2 \sin (50 x), 0,1), & x>0.1\end{cases}$

and the computational domain is $[0,1]$ with prescribed free boundary conditions $(\mathrm{cfl}=0.219$, simulation time $0.18 \mathrm{~s}$, initial number of cells 500 and 4 levels of mesh refinement). As a reference solution, we compute the solution on a uniform fixed grid (20000 cells) with the RK2 scheme. This solution being computed on a very fine fixed grid, as predicted by the theory, the density of entropy production is almost concentrated at the shocks. Even if small productions are present between $0.5 \leq x \leq 0.75$, one can consider such a solution as an "exact" one.

On Figure 1, we plot the density of the reference solution, the one by AB1, AB2 and RK2 schemes and their numerical density of entropy production. Starting from 500 cells, the adaptive schemes lead to very close solutions for each scheme and the numerical density of entropy production vanishes everywhere where the solution is smooth and every solution fit to the reference solution. However, focusing closely to the oscillating area between $0.5 \leq x \leq 0.7$, one can observe that the standard classification of methods holds: AB1, AB2 and RK2. Table 1 

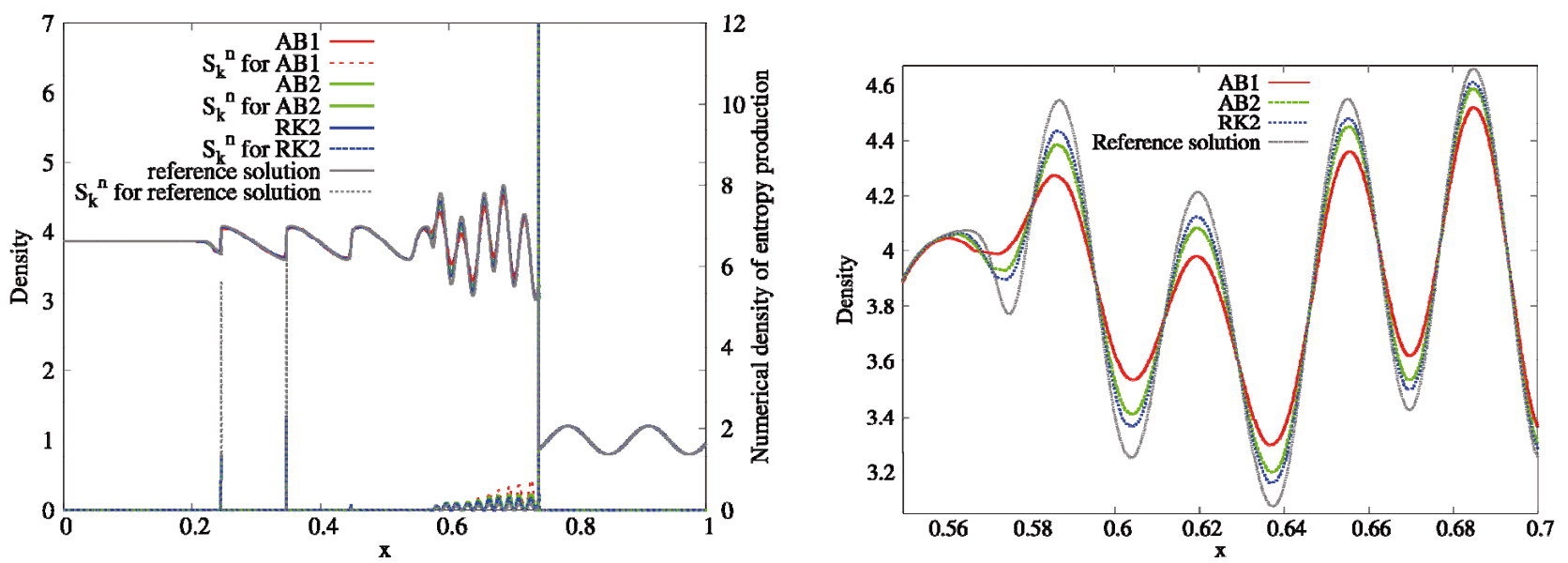

Fig. 1. Shu Osher test case. (a) Density and numerical density of entropy production. (b) Zoom on oscillating region.

summarizes the computation of the total entropy production $\mathcal{P}$ (integral of $(2)$ in time and space), the discrete $l_{x}^{1}$ norm of the error on the density, the cpu-time, the average number of cells and the maximum number of cells at final time. It is well-known that the AB2 scheme is less stable and less accurate than the RK2 scheme. Nonetheless, in the framework of the local time stepping, for almost the same accuracy the AB2M scheme computes 3 times faster than the RK2 which corresponds to a significant gain in time.

\section{Dambreak problem}

We now present one result computed using the isothermal bi-fluid model [11]:

$$
\begin{aligned}
& \frac{\partial \rho}{\partial t}+\operatorname{div}(\rho \vec{u})=0, \quad \frac{\partial \rho \vec{u}}{\partial t}+\operatorname{div}(\rho \vec{u} \otimes \vec{u}+p \overline{\bar{I}})=\rho \vec{g} \\
& \frac{\partial \varphi}{\partial t}+\vec{u} \cdot \nabla \varphi=0 \\
& p=p_{0}+c_{0}^{2}\left[\rho-\left(\varphi \rho_{A}+(1-\varphi) \rho_{W}\right)\right]
\end{aligned}
$$

where $\varphi$ denotes the fraction of water. We define the entropy by

$$
s=\frac{1}{2} \rho u^{2}+c_{0}^{2} \rho \ln (\rho)-c_{0}^{2}\left(\rho_{W}-\rho_{A}\right) \varphi
$$

and the entropy flux by

$$
\begin{aligned}
\psi=\left(s+c_{0}^{2} \rho-c_{0}^{2}\left(\rho_{W}-\rho_{A}\right) \varphi\right) \vec{u} & = \\
& \left(\frac{1}{2} \rho u^{2}+c_{0}^{2} \rho(\ln \rho+1)\right) \vec{u} .
\end{aligned}
$$

Instead of octree meshing, we use cartesian block meshing. The computational domain is splitted in many "blocks" which are devoted to a parallel process. According to the average entropy production and thus the mesh refinement level $N$, each block is meshed in a cartesian way $\left(2^{N-1} n_{x} \times 2^{N-1} n_{y} \times 2^{N-1} n_{z}\right)$ cells. The interface between two blocks is therefore, most of the time, a non conforming one.

The model is applied to the classical dambreaking problem with an obstacle (as described by Koshizuka in [26]). The computational domain $(584 \mathrm{~mm} \times 584 \mathrm{~mm} \times$ $0.5 \mathrm{~mm}$ ) is splitted in 321 blocks which will be dispached on 120 cores. The air-water interface is well described according to the mesh refinement procedure which follows the high values of the numerical production of entropy. Let us denote $p_{e}$ the numerical density of entropy production (2) and $\overline{p_{e}}$ its average value over the domain at time $t$. If the average value of $p_{e}$ over a block is less than $2 \%$ of $\overline{p_{e}}$, the block is coarsened. And if the average value of $p_{e}$ over a block is more than $20 \%$ of $\overline{p_{e}}$, the block is refined. According to [17] the level of two adjacent blocks never exceeds 2 in order to avoid oscillations. Figures 2 (resp. 3) represent the results at $t=0.2 \mathrm{~s}$ (resp. $t=0.4 \mathrm{~s})$. Without any restriction, the maximum number of cells is between 70000 and 100000 during all the simulation for an elapsed computing time about $5 \mathrm{~h}$. In this simulation each block is meshed in a Cartesian way with $2 \times 2^{N-1}$ cells in the $x$ and $y$ direction and always 1 cell in the $z$ direction. It leads to a mesh size from $12 \mathrm{~mm}$ to $0.75 \mathrm{~mm}$ in this example.

\section{Conclusion}

In this paper, we use a first and second order methods in space and time which are coupled with an adaptive algorithm employing local time stepping. In this adaptive numerical scheme the grid is locally refined or coarsened according to the entropy indicator. Several numerical tests have been performed and show an impressive improvement with respect to uniform grids even if a large number of cells is used.

All numerical tests also show that the numerical density of entropy production combined with the proposed mesh refinement parameter is a relevant local error indicator (everywhere where the solution remains smooth) and discontinuity detector: large shocks and oscillating solutions are very well-captured. Moreover, we 
L. Yushchenko et al.: Mechanics \& Industry 16, 301 (2015)
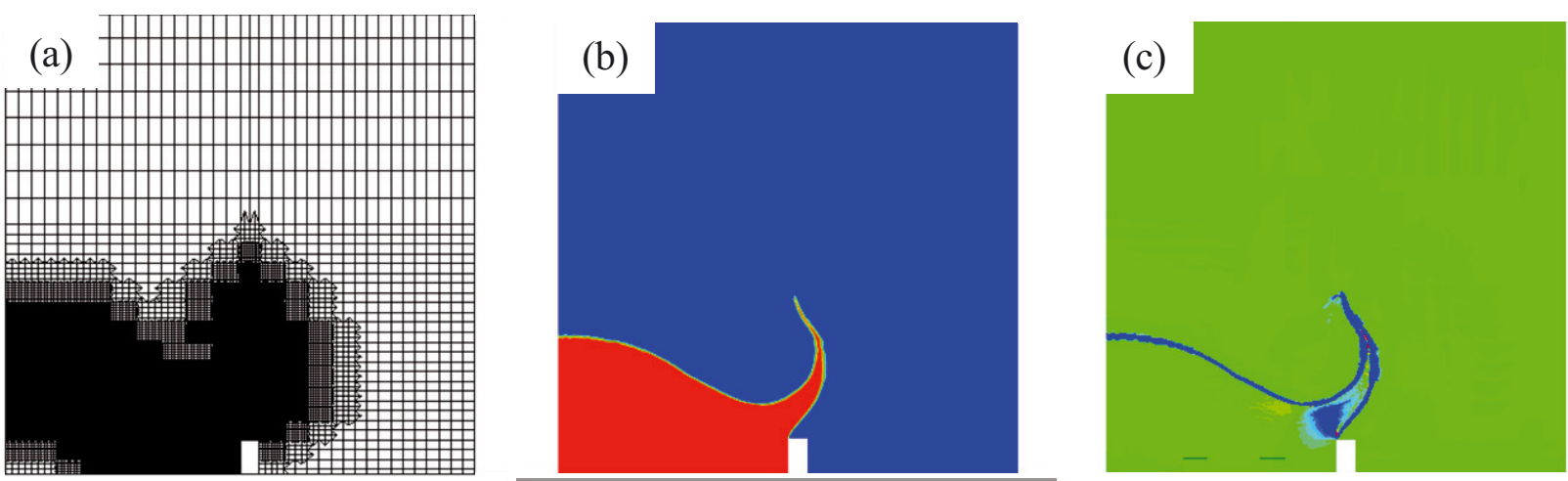

(d)
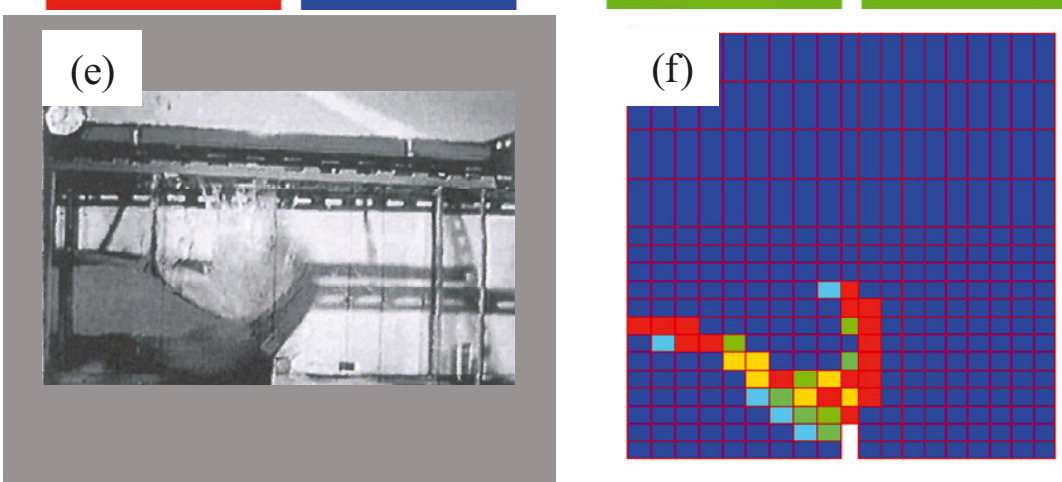

Fig. 2. Dambreak with block remeshing at $t=0.2$ s. (a) Mesh; (b) density (air-blue, water-red); (c) density of numerical entropy production (Eq. (2): green-zero, blue-negative values); (d) mesh refinement level per block (1 to 5); (e) experiment [26]; (f) mesh refinement criterion per block.

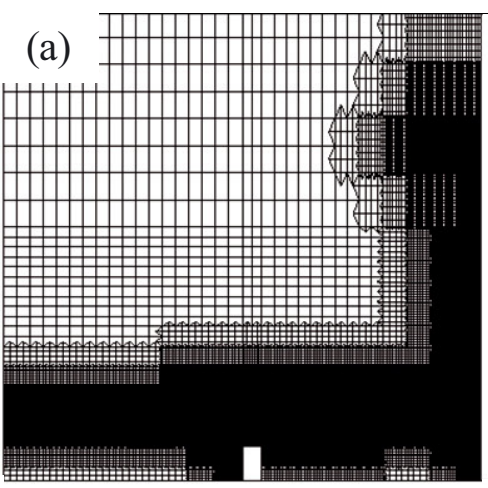

(d)

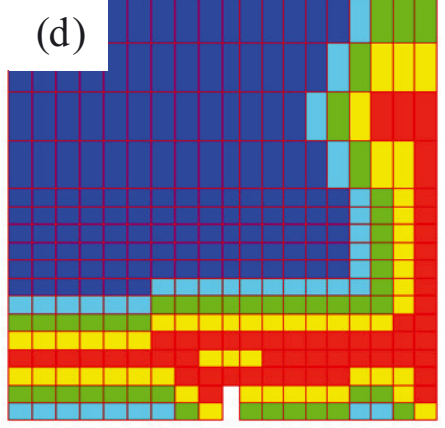

(b)

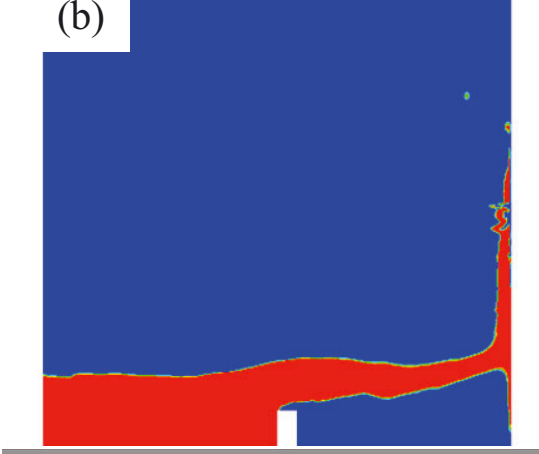

(e)

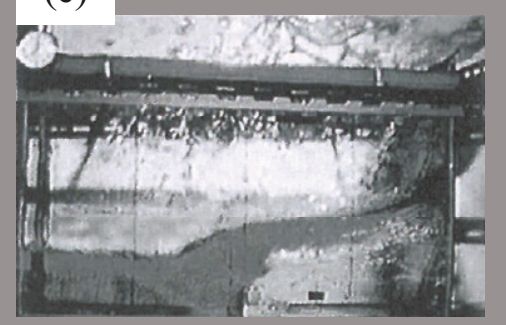

(c)

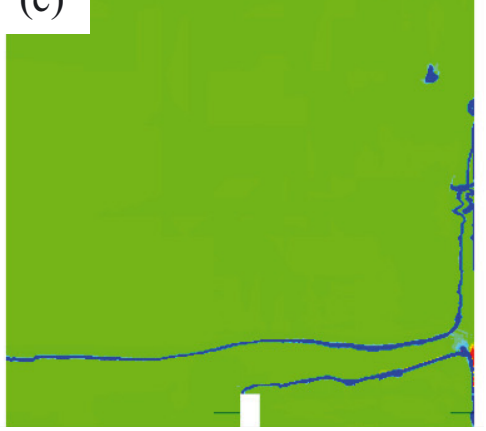

(f)

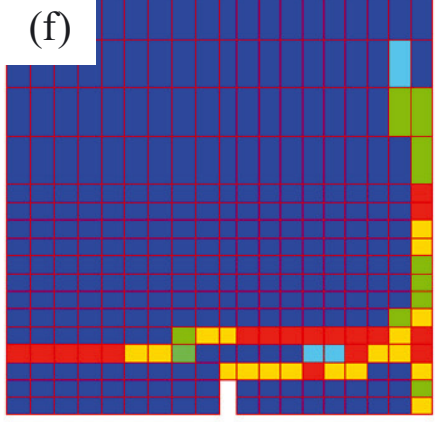

Fig. 3. Dambreak with block remeshing at $t=0.4$ s. (a) Mesh; (b) density (air-blue, water-red); (c) density of numerical entropy production (Eq. (2): green-zero, blue-negative values); (d) Mesh refinement level per block (1 to 5); (e) experiment [26]; (f) mesh refinement criterion per block. 
have shown that the implementation of the local time stepping algorithm can significantly reduce the computational time keeping the same order of accuracy. Applied to complex air-water flows, it is a accurate and powerful numerical tool. This approach can be even improved by balancing more efficiently the tasks in the parallel process.

Acknowledgements. Computations have been performed at the Mésocentre d'Aix-Marseille Université. This work was partially supported by the Grant MTM2011-29306 of the MICINN, Spain, and the ModTerCom project within the APEX program of the region Provence-Alpe-Côte d'Azur.

\section{References}

[1] S. Abadie, D. Morichon, S. Grilli, S. Glockner, Numerical simulation of waves generated by landslides using a multiple-fluid Navier-Stokes model, Coast. Eng. 57 (2010) 779-794

[2] D. Fuster, G. Agbaglah, C. Josserand, S. Popinet, S. Zaleski, Numerical simulation of droplets, bubbles and waves: state of the art, Fluid Dyn. Res. 41 (2009) 065001

[3] S. Vincent, J.P. Caltagirone, P. Lubin, T. Randrianarivelo, An adaptive augmented lagrangian method for three-dimensional multimaterial flows, Comput. Fluids 33 (2004) 1273-1289

[4] K.M.T. Kleefsman, G. Fekken, A.E.P. Veldman, B. Iwanowski, B. Buchner, A volume-of-fluid based simulation method for wave impact problems, J. Comput. Phys. 206 (2005) 363-393

[5] S.C. Hsiao, T.C. Lin, Tsunami-like solitary waves impinging and overtopping an impermeable seawall: Experiment and RANS modeling, Coast. Eng. 57 (2010) 1-18

[6] A. Yeganeh-Bakhtiary, F. Hajivalie, A. Hashemi-Javan, Steady streaming and flow turbulence in front of vertical breakwater with wave overtopping, Appl. Ocean Res. 32 (2010) 91-102

[7] P. Guyenne, S.T. Grilli, Numerical study of threedimensional overturning waves in shallow water, J. Fluid Mech. 547 (2006) 361-388

[8] M.S. Çelebi, Computation of transient nonlinear ship waves using an adaptive algorithm, J. Fluids Struct. 14 (2000) 281-301

[9] D. Dutykh, R. Poncet, F. Dias, The volna code for the numerical modeling of tsunami waves: Generation, propagation and inundation, Eur. J. Mech. B/Fluids 30 (2011) 598-615

[10] P. Helluy, F. Golay, J.P. Caltagirone, P. Lubin, S. Vincent, D. Drevrard, R. Marcer, P. Fraunié, N. Seguin, S. Grilli, A.N. Lesage, A. Dervieux, O. Allain, Numerical simulation of wave breaking, M2AN (2005)
[11] F. Golay, P. Helluy, Numerical schemes for low Mach wave breaking, Int. J. Comput. Fluid Dyn. 21 (2007) 69-86

[12] A. Sambe, F. Golay, D. Sous, P. Fraunié, R. Marcer, Numerical wave breaking over macro-roughness, Eur. J. Mech. B/Fluid 30 (2011) 577-588

[13] F. Golay, Numerical entropy production and error indicator for compressible flows, C.R. Mecanique 337 (2009) 233-237

[14] G. Puppo, Numerical entropy production on shocks and smooth transitions, SIAM, J. Sci. Comput. 17 (2002) 263271

[15] G. Puppo, Numerical entropy production for central schemes, SIAM, J. Sci. Comput. 25 (2003) 1382-1415

[16] G. Puppo, M. Semplice, Numerical entropy and adaptivity for finite volume schemes, Commun. Comput. Phys. 10 (2011) 1132-1160

[17] M. Ersoy, F. Golay, L. Yushchenko, Adaptive multi-scale scheme based on numerical entropy production for conservation laws, Cent. Eur. J. Math. 11 (2013) 1392-1415

[18] M.J. Berger, J. Oliger, Adaptive mesh refinement for hyperbolic partial differential equations, J. Comput. Phys. 53 (1984) 484-512

[19] P. Houston, J.A. Mackenzie, E. Süli, G. Warnecke, A posteriori error analysis for numerical approximations of Friedrichs systems, Num. Math. 82 (1999) 433-470

[20] S. Karni, A. Kurganov, Local error analysis for approximate solutions of hyperbolic conservation laws, Adv. Comput. Math. 22 (2005) 79-99

[21] S. Müller, Y. Stiriba, Fully adaptive multiscale schemes for conservation laws employing locally varying time stepping, J. Sci. Comput. 30 (2007) 493-531

[22] Z. Tan, Z. Zhang, Y. Huang, T. Tang, Moving mesh methods with locally varying time steps, J. Comput. Phys. 200 (2004) $347-367$

[23] C. Altmann, T. Belat, M. Gutnic, P. Helluy, H. Mathis, E. Sonnendrücker, W. Angulo, J.M. Hérard, A local timestepping discontinuous Galerkin algorithm for the MHD system, ESAIM 28 (2009) 33-54

[24] S. Osher, R. Sanders, Numerical approximations to nonlinear conservation laws with locally varying time and space grids, Math. Comput. 41 (1983) 321-336

[25] C.W. Shu, S. Osher, Efficient implementation of essentially nonoscillatory shock-capturing schemes, J. Comput. Phys. 77 (1988) 439-471

[26] S. Koshizuka, H. Tamako, Y. Oka, A particle method for incompressible viscous flow with fluid fragmentations, Comput. Fluid Dyn. J. 4 (1995) 29-46

[27] S. Popinet, Gerris: a tree-based adaptive solver for the incompressible Euler equations in complex geometries, J. Comput. Phys. 190 (2003) 572-600

[28] S. Popinet, G. Rickard, A tree-based solver for adaptive ocean modeling, Ocean Mod. 16 (2007) 224-249 